\title{
Clinical Characteristics and Current Medical Practice in a Group of Sudanese Patients with Epilepsy: A Cross Sectional Hospital Based-Study
}

\author{
Muaz Elsayed 1,2,3,4*, Mohamed Issa ${ }^{2}$, Ibrahim Mahmoud ${ }^{3}$, Sarah Imam ${ }^{3,4}$ \\ ${ }^{1}$ College of Medicine, Omdurman Islamic University, Omdurman, Khartoum, Sudan \\ ${ }^{2}$ Omdurman Teaching Hospital, Omdurman, Khartoum, Sudan \\ ${ }^{3}$ College of Medicine, University of Sharjah, Sharjah, UAE \\ ${ }^{4}$ University Hospital of Sharjah, Sharjah, UAE \\ Email: ^muaz_muaz@hotmail.com,mima8007@gmail.com, iabdelmahmoud@sharjah.ae.ac, Sharjah.sarah.mohamed@uhs.ae
}

How to cite this paper: Elsayed, M., Issa, M., Mahmoud, I. and Imam, S. (2021) Clinical Characteristics and Current Medical Practice in a Group of Sudanese Patients with Epilepsy: A Cross Sectional Hospital Based-Study. Case Reports in Clinical Medicine, 10, 264-276. https://doi.org/10.4236/crcm.2021.1010034

Received: August 25, 2021

Accepted: October 10, 2021

Published: October 13, 2021

Copyright $\odot 2021$ by author(s) and Scientific Research Publishing Inc. This work is licensed under the Creative Commons Attribution International License (CC BY 4.0).

http://creativecommons.org/licenses/by/4.0/

\section{Abstract}

Introduction: The epilepsy classification in under developed countries faces many difficulties in reporting, work-up and management strategies. Exploring local practice in general hospitals will positively add to the welfare of patients with epilepsy. The objectives of this study were to assess the current medical practice in epilepsy work up and to study the selection of AEDs as per ILAE guidelines. Methods: This was a cross sectional-retrospective hospital based study, conducted between April and September 2016 in Omdurman Teaching Hospital, Khartoum, Sudan. Patients aged 18 years old and above were enrolled. Epilepsy was defined as having at least two non-provoked seizures in the least 6 months in a patient who was assessed by clinical review and electroencephalogram (EEG). Epilepsy was classified as generalized, focal or unclassified. Medications refer to all internationally licensed antiepileptic medications (AEDs) in 2016. Results: One hundred adult Sudanese patients were enrolled for this study. The most common event described during the ictal phase was tongue biting in $50 \%$ of participants followed by body stiffness in $46 \%$. Epilepsy was classified as generalized in $84 \%$, focal in $11 \%$ and unclassified in 5\% patients. In generalized epilepsy, the MRI detected $23.3 \%$ abnormal findings higher than the CT which detected $14.8 \%(4 / 27), \mathrm{p}$ value $=$ 0.032. In focal epilepsy, the CT detected $75 \%$ abnormal findings higher than the MRI which detected $33.3 \%$, p value $=0.02$. The AEDs used were as follows: Carbamazepine (CBZ) 48\%, Na valproate (VP) 33\%, Lamotrigine (LMT) $2 \%$, Levetricetan (LVT) 1\%, CBZ + VP 14\% and CBZ + Oxcarbaze- 
pine (OXC) 2\%. Conclusion: The current medical practice in Omdurman teaching hospital should be modified to match the international league against epilepsy (ILAE) guidelines in workup, management, AEDs selection and classification of epilepsy.

\section{Keywords}

Anti-Epileptic Drugs, EEG, Epilepsy, Sudan

\section{Introduction}

Epilepsy is considered one of the most prevalent neurological disorders worldwide affecting over 45 million people [1]. The disease has a huge burden in the developing countries as over $80 \%$ of cases occurring in low- and middle-income countries [2] [3]. In Sub-Saharan Africa including Sudan, the prevalence of epilepsy was estimated to be 9 per 1000 persons [4].

The workup of epilepsy for the benefit of safe diagnosis and management options may differ due to the healthcare system criteria and many local, social, financial or environmental factors. The mixture of all those factors determines the strategy behind epilepsy management which may not be met by the international recommendations particularly in fragile healthcare systems.

Variations in prevalence of different types of epilepsy have been reported to vary across regions [5] [6] [7] [8]. In Sudan, the prevalence of generalized epilepsy was estimated to be $56 \%$ followed by focal epilepsy $36 \%$ and $8 \%$ for unclassified epilepsy [9]. The electroencephalography (EEG) is the main tool for potentiating the diagnosis, classification and prognostic criteria for epilepsy. It has a moderate sensitivity and a high specificity [10]. Further recording may increase the likelihood but around 10\% - 40\% may not show abnormalities on a routine EEG [11]. The recording in the first 24 hours after the seizure has a higher yield. Combining modern computer techniques, Magnetoencephalography (M/EEG), and in depth EEG may increase the yield of the EEG in classification of epilepsy [12]. The superiority of the magnetic resonance imaging (MRI) scans had bridged a large gap in the localization related epilepsies. A large study across 14 countries concluded that the MRI was the preferred imaging tool to detect cortical and subcortical changes in epilepsy [13].

The therapeutic paradigm of epilepsy should consider the rational selection of anti-epileptic drug (AED) in relation to the cause and classification of epilepsy. In addition, other factors are playing a major role in determining the selection process such as professional competency, location, and socio-economic status [14]. Generally, around 70\% of epilepsy patients can be fairly controlled with medications and life style changes [15]. The persistent effort of the treating doctor to control intractable patients may be achieved in some through thoughtful AED changes [16]. 
The epidemiological features, workup and diagnostic accuracy might differ across countries and regions. The aim of this study was to examine the current workup for epilepsy in medical practice and selection of AEDsa tertiary hospital in Sudan. Specifically, the study determined the diagnostic approach for epilepsy, verified the selection of AEDs in relation to seizure semiology and EEG findings, and evaluated the current practice of clinicians in the selection of AEDs.

\section{Methods}

This was a cross sectional-retrospective hospital based study conducted between April to September 2016 in Omdurman Teaching Hospital Khartoum Sudan. It is the second central hospital in the country. Adult Sudanese patients above the age of 18 years old and above were included after their consent. Patients presenting either to the emergency room or outpatient department were included. Their diagnosis was confirmed by history, examination, imaging and EEG. All patients were on anti-epileptic drugs (AEDs) for at least 6 months. Patients with an uncertain diagnosis or symptomatic seizures were excluded. The study sample was collected through total coverage sampling technique. The sample size was 100 patients representing complete coverage. Epilepsy was defined as having at least two non-provoked seizures for in the least 6 months in a patient who was assessed by clinical review and EEG. Antiepileptic medications refer to all internationally licensed AEDs in 2016. The EEG represented the standard 16 channel EEG recording for at least 20 minutes or more with photic stimulation and hyperventilation techniques. Seizures were defined as generalized, partial, complex partial and secondary generalized and epilepsy was classified as generalized or focal. Information was collected using a questionnaire containing personal data, important history points in regard to the symptomatology, diagnosis, EEG report and the details of AEDs that were used. The questionnaire was validated with Cronbach's alpha of 86 .

\subsection{Ethical Approval}

Institutional ethical committee approval was obtained prior the start of study and informed consent was taken from all the study participants.

\subsection{Statistical Analysis}

Mean (standard deviations, SD) were reported for numerical variables. Frequencies with percentages were reported for categorical variables. Pearson Chi squares test was used to examine the association between categorical variables. Statistical significance was set at $\mathrm{p}<0.05$.

Analysis was performed using Statistical Package for Social Science (SPSS), version 23 (IBM, Armonk, NY, USA).

\section{Results}

Males were found to be $52 \%$ with a mean age of the entire participants was 31.5 
years (standard deviation \pm 15.3 years).

Table 1 shows types and clinical characteristics of epilepsy. In this study the symptomatic description of the seizure was $84 \%$ generalized, $11 \%$ focal and $5 \%$ unclassified. Visual aura was reported in $20 \%$, somatosensory aura in $10 \%$, abdominal aura $8 \%$, psychic aura $7 \%$, autonomic aura $5 \%$ and olfactory aura $3 \%$. The most common events described during the ictal phase was tongue biting in $50 \%$ of participants followed by body stiffness in $46 \%$. In $37 \%$ there was collapse and loss of body posture while $30 \%$ had myoclonic jerks. Incontinence of sphincters was in $20 \%, 10 \%$ were hypotonic, $10 \%$ had lip smacking, chewing movements were in $6 \%$ and $4 \%$ had undressing. The duration of the seizure was described to be $<2$ minutes in $48 \%$, more than that in $47 \%$ and $5 \%$ had last for

Table 1. Types and clinical characteristics of epilepsy.

\begin{tabular}{|c|c|c|}
\hline Variables & Percentages \% & Number of patients \\
\hline \multicolumn{3}{|l|}{ Type of auras } \\
\hline Visual & 20 & 20 \\
\hline Somatosensory & 10 & 10 \\
\hline Abdominal & 8 & 8 \\
\hline Psychic & 7 & 7 \\
\hline Autonomic & 5 & 5 \\
\hline Olfactory & 3 & 3 \\
\hline \multicolumn{3}{|l|}{ Types of epilepsy } \\
\hline Generalized & 84 & 84 \\
\hline Focal & 11 & 11 \\
\hline Unclassified & 5 & 5 \\
\hline \multicolumn{3}{|c|}{ Duration of seizure, minutes } \\
\hline$<1$ & 5 & 5 \\
\hline $1-2$ & 48 & 48 \\
\hline$>2$ & 47 & 47 \\
\hline \multicolumn{3}{|c|}{ Events during the ictal phase } \\
\hline Tongue biting & 50 & 50 \\
\hline Body stiffness & 46 & 46 \\
\hline Collapsed & 37 & 37 \\
\hline Myoclonic jerks & 30 & 30 \\
\hline Incontinence & 20 & 20 \\
\hline Hypotonia & 10 & 10 \\
\hline Lip smacking & 10 & 10 \\
\hline Chewing & 6 & 6 \\
\hline Undressing & 4 & 4 \\
\hline \multicolumn{3}{|l|}{ Neurological examination } \\
\hline Normal & 78 & 78 \\
\hline Focal signs & 22 & 22 \\
\hline
\end{tabular}


few seconds. The majority of patients had normal neurological examination (78\%), while $22 \%$ had focal neurological signs either old or new (Table 1 ).

Distribution of the patients according to neuroimaging (CT/MRI) is shown in Table 2. From the total enrolled patients, a brain MRI was performed in $46 \%$ CT brain was done in $31 \%$, both MRI and CT were done in $6 \%$ and $23 \%$ of patients could not afford a brain image. In generalized epilepsy, the MRI detected 23.3\% (10/43) abnormal findings higher than the CT which detected 14.8\% (4/27), p value $=0.032$. In focal epilepsy, the CT detected $75 \%$ (3/7) abnormal findings higher than the MRI which detected 33.3\% (1/8), p value $=0.021$ (Table 2).

Table 3 shows the distribution of the patients according to the electroencephalogram (EEG) findings by their clinical diagnosis and epilepsy type. Generalized epileptiform discharge was found in $22 \%$, focal epileptic form in $12 \%$, abnormal findings in $4 \%$ and normal findings in $16 \%$ of the patients. The patients who were diagnosed clinically as generalized epilepsy and their EEG confirmed the diagnosis were $37.5 \%$, while $20.8 \%$ of those patients their EEG showed focal epileptic form discharge, $\mathrm{p}$ value $=0.011$. The patients who were diagnosed clinically as focal epilepsy and their EEG confirmed the diagnosis were $40 \%$, equal to those patients their EEG showed generalized epileptic form discharge, $\mathrm{p}$ value $=$ 0.930 .

Table 4 shows the types of anti-epileptic drugs (AEDs) used in this study and their prescribed total daily doses. The AEDs used in this group of patients were

Table 2. Brain imaging stratified by types of epilepsy.

\begin{tabular}{|c|c|c|c|c|}
\hline \multirow{2}{*}{ Brain Imaging } & \multirow{2}{*}{ Findings } & \multicolumn{2}{|c|}{ Generalized Epilepsy } & \multirow{2}{*}{$P$ valu } \\
\hline & & $\mathbf{N}$ & $\%$ & \\
\hline \multirow{4}{*}{ MRI } & Normal & 33 & 76.7 & \multirow{8}{*}{0.032} \\
\hline & Abnormal & 10 & 23.3 & \\
\hline & Total & 43 & 100.0 & \\
\hline & Not done & 45 & 51.1 & \\
\hline \multirow{4}{*}{$\mathrm{CT}$} & Normal & 23 & 85.2 & \\
\hline & Abnormal & 4 & 14.8 & \\
\hline & Total & 27 & 100.0 & \\
\hline & Not done & 61 & 69.3 & \\
\hline \multicolumn{5}{|c|}{ Focal Epilepsy } \\
\hline \multirow{4}{*}{ MRI } & Normal & 2 & 66.7 & \multirow{8}{*}{0.021} \\
\hline & Abnormal & 1 & 33.3 & \\
\hline & Total & 3 & 100 & \\
\hline & Not done & 8 & 72.7 & \\
\hline \multirow{4}{*}{ CT } & Normal & 1 & 25 & \\
\hline & Abnormal & 3 & 75 & \\
\hline & Total & 4 & 100 & \\
\hline & Not done & 7 & 63.6 & \\
\hline
\end{tabular}


Table 3. Electroencephalogram (EEG) findings stratified by clinical diagnosis and types of epilepsy.

\begin{tabular}{|c|c|c|c|c|c|}
\hline \multirow{3}{*}{ EEG finding } & \multicolumn{4}{|c|}{ Generalized Epilepsy (semiology) } & \multirow{3}{*}{$P$ value } \\
\hline & \multicolumn{2}{|c|}{ Yes } & \multicolumn{2}{|c|}{ No } & \\
\hline & $\mathrm{N}$ & $\%$ & $\mathbf{N}$ & $\%$ & \\
\hline Normal & 15 & 31 & 1 & 13 & \\
\hline Generalized epileptiform discharge & 18 & 38 & 4 & 50 & \\
\hline Focal epileptiform discharge & 10 & 21 & 2 & 25 & 0011 \\
\hline Abnormal EEG & 5 & 10 & 1 & 13 & \\
\hline Total & 48 & 100 & 8 & 100 & \\
\hline \multirow[t]{2}{*}{ Not done } & 40 & 46 & 8 & 50 & \\
\hline & \multicolumn{4}{|c|}{ Focal Epilepsy (semiology) } & \\
\hline Normal & 0 & 0.0 & 16 & 33 & \multirow{6}{*}{0.930} \\
\hline Generalized epileptiform discharge & 2 & 40.0 & 20 & 41 & \\
\hline Focal epileptic discharge & 2 & 40.0 & 10 & 20 & \\
\hline Abnormal EEG & 1 & 20 & 3 & 6 & \\
\hline Total & 5 & 100 & 49 & 100 & \\
\hline Not done & 6 & 55 & 40 & 82 & \\
\hline
\end{tabular}

Table 4. Antiepileptic drugs (AED) stratified by daily doses.

\begin{tabular}{|c|c|c|c|}
\hline AED & Daily Dose/mg & $\mathbf{N}$ & $\%$ \\
\hline \multirow[t]{5}{*}{ Carbamazepine } & 200 & 3 & 2 \\
\hline & 400 & 22 & 22 \\
\hline & 600 & 17 & 15 \\
\hline & 800 & 3 & 3 \\
\hline & 1200 & 3 & 3 \\
\hline \multirow[t]{6}{*}{ Na valproate } & 200 & 6 & 6 \\
\hline & 400 & 12 & 11 \\
\hline & 600 & 5 & 5 \\
\hline & 800 & 6 & 6 \\
\hline & 1000 & 2 & 2 \\
\hline & 1500 & 2 & 2 \\
\hline Lamotrigine & 100 & 2 & 2 \\
\hline Levetricetan & 250 & 1 & 1 \\
\hline Carbamazepine $+\mathrm{Na}$ valproate & $200 / 400$ & 14 & 14 \\
\hline Oxcarbazepine + Carbamazepine & $400 / 600$ & 2 & 2 \\
\hline Total & & 100 & 100 \\
\hline
\end{tabular}

as follows: Carbamazepine (CBZ) 48\%, Na valproate (VP) 33\%, Lamotrigine (LMT) $2 \%$, Levetricetan (LVT) 1\%, CBZ + VP 14\% and CBZ + Oxcarbazepine (OXC) $2 \%$.

The management of the patients based on clinical grounds shows that patients 
diagnosed as generalized epilepsy received were mainly received CBZ 48.9\% (43/88) and VP 33\% (29/88) almost similar to patients diagnosed as focal epilepsy who received CBZ 41.7\% (5/12) and VP 33.3\% (4/12), (Table 5). Combinations of CBZ \& VP were prescribed for $13.6 \%(12 / 88)$ with generalized epilepsy and for $16.7 \%$ (2/12) with focal epilepsy (Table 5).

\section{Discussion}

Our findings in semiology of generalized seizures (84\%) was higher than a hospital based study in Iran (78\%) [17] and also higher than the French and Spain studies (31\% \& 13.8\%), in hospital and primary care setting respectively [18] [19]. The tonic clonic seizures here was $75 \%$ which was partially matching a previous study in the same hospital which was $72.3 \%$ [20]. The differences in design and methodologies had affected our hospital based results. The common semiology's which helped to diagnose epilepsy in this study were tongue biting in $50 \%$, body stiffness in $48 \%$ and collapse in $37 \%$. All patients had a usual duration of events that last between few seconds to maximum 2 minutes for a single event. This is well convenient with the natural history of seizures.

The type of epilepsy classified as generalized epilepsy (61\%) in this study did not match a previous study in Omdurman teaching hospital (38\%). However, in the later study all patients have EEG while in our patients only 55\% had EEG access. The focal epilepsy partially matched the previous study as it was $26 \%$ versus $29 \%[20]$.

The management of patient's resources in under developed countries affects the management decisions including imaging selection. The Brain MRI in the

Table 5. Antiepileptic drugs (AEDs) stratified by types of epilepsy.

\begin{tabular}{lcc}
\hline \multirow{2}{*}{ AED } & \multicolumn{2}{c}{ Generalized epilepsy } \\
\cline { 2 - 3 } & N & $\%$ \\
\hline Carbamazepine & 43 & 49 \\
Na valproate & 29 & 33 \\
Lamotrigine & 2 & 2 \\
Levetiracetam & 1 & 1 \\
Carbamazepine + Na valproate & 12 & 14 \\
Oxcarbazepine + Carbamazepine & 1 & 1 \\
Total & 88 & 100 \\
\hline & & Focal epilepsy \\
\hline Carbamazepine & 5 & 42 \\
Na valproate & 4 & 33 \\
Lamotrigine & 0 & 0 \\
Levetiracetam & 0 & 0 \\
Carbamazepine + Na valproate & 2 & 17 \\
Oxcarbazepine + Carbamazepine & 1 & 8 \\
Total & 12 & 100 \\
\hline
\end{tabular}


enrolled patients was significantly more cost effective (8\%) in detecting lesional epilepsy than CT Brain (3.5\%) even in patients thought to have Idiopathic epilepsy. This had added to epilepsy classification.

The statistical significance of imaging findings between the MRI \& CT Brain in this study conforms to the literature recommendation about the superiority of MRI scans against CT in imaging of epilepsy patients [21] [22]. Moreover, the MRI was the preferred tool the ENIGMA-Epilepsy consortium [13].

The electroencephalography was normal in $16 \%$ of our patients in comparison to $29 \%$ in a larger multi center Iranian study [17]. As known about the increased sensitivity of 3 EEGs, it worth mentioning here that our patients had one EEG during the time of this study. Moreover, the poor significance of the clinical impression in classification of epilepsy was revealed here as $20 \%$ of the patients assumed to have idiopathic generalized epilepsy were relay having generalized epileptiform discharges on their EEGs. In $11 \%$ of the assumed cases of generalized epilepsy had focal discharges altering their epilepsy classification.

On the other hand, $18 \%$ of the clinically assumed focal epilepsy had focal discharges while $18 \%$ of the assumed focal epilepsy had generalized epileptiform discharges on EEG, again altering their epilepsy classification and management.

So the likelihood of having a wrong clinical judgment on epilepsy classification without performing an EEG is higher in focal epilepsy than generalized epilepsy.

The total percentage of patients finally classified as a generalized form of epilepsy was $38 \%$ versus $29 \%$ for focal epilepsy. This is slightly higher in the Market scan data study but the difference is related to sample size, EEG technique, age, demographic features and accuracy of epidemiological studies [23].

Our epilepsy classification figures are quite different from the hospital study that targeted elderly population including brain tumors and stroke. The generalized epilepsy was found in $65 \%$ and focal was only $13 \%$ [24]. This can be explained by the young age in our study.

In our sample, the IGE percentage was approaching the percentage of a retrospective tertiary hospital study which was 33\% [25]. However, EEG was applied for all patients and some had multiple EEGs.

Our data were different from the Egyptian school children study. However, the difference is due to differences in design, target population and $49 \%$ in the Egyptian study were diagnosed with specific epileptic syndromes where we expect multiple seizure types in this group of patients [25].

This essential part of the study matched a research question of the authors that the selection for AEDs was not suitable in respect to the type of epilepsy and EEG diagnosis. It was clearly found that CBZ was used as major choice in almost half of patients regardless of having focal or generalized epilepsy. This is not suitable as CBZ is licensed for focal epilepsy and it is a second line drug for generalized seizures. However, CBZ was reported as the second used AED (18.7\%) for generalized epilepsy in the Northern Iran study while VLP was the first 
choice as $27.7 \%$ were using it [17]. One of the interesting observations was the unsuitable use of combinations of CBZ and OXC which is not recommended depending on the indications, target receptor and side effects. The use of CBZ in our study, partially matched a previous primary care study which reported it as the second choice [19]. In one study addressing only idiopathic generalized epilepsy, LMT was prescribed in $3 \%$ of the cases and this conforms to our findings [25].

Hence, we observed a significant practice gap noticed by the less use of LMT in generalized epilepsy and more use of CBZ for generalized epilepsy. The same concept was observed in the use of LVT for generalized epilepsy despite the small number of patients suing LVT due to cost and availability issues.

The observed selection bias of AEDs reflects the suboptimal knowledge about epilepsy classification, selection mechanism, drug action and potential side effects. Despite the percentage was small here but it necessitates more target oriented educational effort to be taken by the clinical pharmacists and neurologists to modulate this practice. The current practice needs to be improved by the local application of international guidelines of diagnosis, EEG and selection of AEDs. The ILAE classification of seizures and epilepsy should be made more available and accessible to physicians talking care of epilepsy patients in the under developed world.

This paper comes as a closing workfor 2 previous papers in the same hospital addressing precipitating factors of seizures and adherence to medications in the same hospital. However, it also faced some limitations. The limitation of this study is being that it was hospital based and was performed in a single tertiary center. Moreover, the sample size is relatively small for quite a common neurological disease. The EEG service was not manageable for $45 \%$ of participants which impaired the goal of the $3^{\text {rd }}$ minor objective. There was no follow up to determine emergence of other seizure semiology which may have affected the initial impression about the seizure type.

\section{Conclusion}

The clinical diagnoses of epilepsy type should be potentiated by the electro-encephalography to guide the best epilepsy classification. The EEG has good sensitivity for generalized epilepsy in patients who were suspected to have generalized epilepsy in this study group in contrast to mildly lower sensitivity for patients clinically suspected to have focal epilepsy. The EEG has high positive predictive value in partial epilepsy diagnosis for this group. The MRI scan had a higher predictive value in epilepsy patients. Selection of AEDs for the type of epilepsy in our hospital medical practice is not matching the ILAE recommendations which are reflected as unsatisfactory selection of AED. More structured awareness is needed to raise the knowledge and modify medical practice, verify semiology, EEG importance and selection of AEDs to help the wellbeing of epilepsy patients in this area. Collaboration of the related local and international bodies will help 
to improve this situation.

\section{Acknowledgements}

Authors appreciate the Omdurman teaching hospital for supporting this study.

\section{Authors Contributions}

Thee corresponding author contributed to the abstract, introduction, discussion and refrences sections. Dr. Mohamed Issa collected the data and initial references. Dr. Ibrahim Mahmoud contributed to re-writing of methodology and results plus updating references. Dr. SarahIman had edited the manuscript and revised the introduction and discussion.

\section{Conflicts of Interest}

Authors declare no conflict of interest.

\section{References}

[1] GBD 2016 Epilepsy Collaborators (2019) Global, Regional, and National Burden of Epilepsy, 1990-2016: A Systematic Analysis for the Global Burden of Disease Study 2016. The Lancet Neurology, 18, 357-375. https://doi.org/10.1016/S1474-4422(18)30454-X

[2] Meyer, A.C., Dua, T., Ma, J., Saxena, S. and Birbeck, G. (2010) Global Disparities in the Epilepsy Treatment Gap: A Systematic Review. Bulletin of the World Health Organization, 88, 260-266. https://doi.org/10.2471/BLT.09.064147

[3] Bharucha, N., Carpio, A. and Gallo Diop, A. (2008) Epidemiology of Epilepsy in Developing Countries. In: Engel, P. and Pedley, T., Eds., Epilepsy: A Comprehensive Textbook, 2nd Edition, Lippincott Williams \& Wilkins, Philadelphia, 89-101.

[4] Owolabi, L.F., Adamu, B., Jibo, A.M., Owolabi, S.D., Isa, A.I., Alhaji, I.D., et al. (2020) Prevalence of Active Epilepsy, Lifetime Epilepsy Prevalence, and Burden of Epilepsy in Sub-Saharan Africa from Meta-Analysis of Door-to-Door Population-Based Surveys. Epilepsy \& Behavior, 103, Article ID: 106846. https://doi.org/10.1016/j.yebeh.2019.106846

[5] Beghi, E. (2020) The Epidemiology of Epilepsy. Neuroepidemiology, 54, 185-191. https://doi.org/10.1159/000503831

[6] Pakdaman, H., Harandi, A.A., Gharagozli, K., Alaeddini, F., Esfandani, A., Seyed Mirbehbahani, H., et al. (2021) Epilepsy Lifetime Prevalence in Iran: A Large Population-Based National Survey. Scientific Reports, 11, Article No. 9437. https://doi.org/10.1038/s41598-021-89048-Z

[7] Song, P., Liu, Y., Yu, X., Wu, J., Poon, A.N., Demaio, A., et al. (2017) Prevalence of Epilepsy in China between 1990 and 2015: A Systematic Review and Meta-Analysis. Journal of Global Health, 7, Article ID: 020706. https://doi.org/10.7189/jogh.07.020706

[8] Alshahawy, A.K., Darwish, A.H., Elsaid Shalaby, S. and Mawlana, W. (2018) Prevalence of Idiopathic Epilepsy among School Children in Gharbia Governorate, Egypt. Brain \& Development, 40, 278-286. https://doi.org/10.1016/j.braindev.2017.12.009

[9] Mohamed, I.N., Elseed, M.A., Hamed, A.A., Abdel-Rahman, M.E., El-Sadig, S.M., Omer, I.M., et al. (2017) Prevalence of Epilepsy in 74,949 School Children in Khar- 
toum State, Sudan. Paediatrics and International Child Health, 37, 188-192. https://doi.org/10.1080/20469047.2016.1278110

[10] Smith, S.J.M. (2005) EEG in the Diagnosis, Classification, and Management of Patients with Epilepsy. Journal of Neurology, Neurosurgery \& Psychiatry, 76, ii2-ii7. https://doi.org/10.1136/jnnp.2005.069245

[11] Baumgartner, C. and Koren, J.P. (2018) Seizure Detection Using Scalp-EEG. Epilepsia, 59, 14-22. https://doi.org/10.1111/epi.14052

[12] Koster, I., Ossenblok, P., Brekelmans, G.J., van der Linden, I., Hillebrand, A., Wijnen, B.F.M., et al. (2020) Sensitivity of Magnetoencephalography as a Diagnostic Tool for Epilepsy: A Prospective Study. Epileptic Disorders, 22, 264-272. https://doi.org/10.1684/epd.2020.1160

[13] Whelan, C.D., Altmann, A., Botia, J.A., Jahanshad, N., Hibar, D.P., Absil, J., et al. (2018) Structural Brain Abnormalities in the Common Epilepsies Assessed in a Worldwide ENIGMA Study. Brain, 141, 391-408. https://doi.org/10.1093/brain/awx341

[14] Shih, J.J., Whitlock, J.B., Chimato, N., Vargas, E., Karceski, S.C. and Frank, R.D. (2017) Epilepsy Treatment in Adults and Adolescents: Expert Opinion, 2016. Epilepsy \& Behavior, 69, 186-222. https://doi.org/10.1016/j.yebeh.2016.11.018

[15] Luciano, A.L. and Shorvon, S.D. (2007) Results of Treatment Changes in Patients with Apparently Drug-Resistant Chronic Epilepsy. Annals of Neurology, 62, 375-381. https://doi.org/10.1002/ana.21064

[16] Neligan, A., Bell, G.S., Elsayed, M., Sander, J.W. and Shorvon, S.D. (2012) Treatment Changes in a Cohort of People with Apparently Drug-Resistant Epilepsy: An Extended Follow-Up. Journal of Neurology, Neurosurgery \& Psychiatry, 83, 810-813. https://doi.org/10.1136/jnnp-2011-302085

[17] Rezaeian Yazdi, M., Mazloum Farsi Baf, M., Afsari, A., Alipour, A., Khorashadizadeh, M., KhajehGhiassi, P., et al. (2015) Clinical Features of Epilepsy at 2 Referral Hospitals in Northern Iran. Neurosciences, 20, 243-247. https://doi.org/10.17712/nsj.2015.3.20140491

[18] Picot, M.C., Baldy-Moulinier, M., Daures, J.P., Dujols, P. and Crespel, A. (2008) The Prevalence of Epilepsy and Pharmacoresistant Epilepsy in Adults: A Population-Based Study in a Western European Country. Epilepsia, 49, 1230-1238. https://doi.org/10.1111/j.1528-1167.2008.01579.x

[19] Fernández-Suárez, E., Villa-Estébanez, R., Garcia-Martinez, A., Fidalgo-González, J.A., Zanabili Al-Sibbai, A.A. and Salas-Puig, J. (2015) Prevalence, Type of Epilepsy and Use of Antiepileptic Drugs in Primary Care. Revista de neurologia, 60, 535-542. https://doi.org/10.33588/rn.6012.2015038

[20] Elsayed, M.A., El-Sayed, N.M., Badi, S. and Ahmed, M.H. (2019) Factors Affecting Adherence to Antiepileptic Medications among Sudanese Individuals with Epilepsy: A Cross-Sectional Survey. Journal of Family Medicine and Primary Care, 8, 2312-2317. https://doi.org/10.4103/jfmpc.jfmpc_405_19

[21] Cendes, F. (2013) Neuroimaging in Investigation of Patients with Epilepsy. Continuum, 19, 623-642. https://doi.org/10.1212/01.CON.0000431379.29065.d3

[22] Cendes, F., Theodore, W.H., Brinkmann, B.H., Sulc, V. and Cascino, G.D. (2016) Neuroimaging of Epilepsy. Handbook of Clinical Neurology, 136, 985-1014. https://doi.org/10.1016/B978-0-444-53486-6.00051-X

[23] Sapkota, S., Kobau, R., Pastula, D.M. and Zack, M.M. (2018) People with Epilepsy Are Diagnosed Most Often with Unspecified Epilepsy, Followed by Focal Epilepsy, Generalized Convulsive Epilepsy, and Generalized Nonconvulsive Epilepsy-US Mar- 
ketScan Data, 2010-2015. Epilepsy \& Behavior, 79, 244-246.

https://doi.org/10.1016/j.yebeh.2017.11.004

[24] Hernandez-Ronquillo, L., Adams, S., Ballendine, S. and Tellez-Zenteno, J.F. (2018) Epilepsy in an Elderly Population: Classification, Etiology and Drug Resistance. Epilepsy Research, 140, 90-94. https://doi.org/10.1016/j.eplepsyres.2017.12.016

[25] Betting, L.E., Mory, S.B., Lopes-Cendes, I., Li, L.M., Guerreiro, M.M., Guerreiro, C.A., et al. (2006) EEG Features in Idiopathic Generalized Epilepsy: Clues to Diagnosis. Epilepsia, 47, 523-528. https://doi.org/10.1111/j.1528-1167.2006.00462.x 


\section{Questionnaire}

A-Personal data:

- NAME.

- AGE

- Gender: Male

Female

- Residence.

- Occupation.

- Contact phone number

B-Type of seizure: Generalized

Focal

C-Seizure semiology:

Precipitating factor: Tiredness

\section{Hunger}

Insomnia $\square \quad$ non-adherence

Current infection

Mood change: Excited $\square \quad$ Anxious

Depressed

Sounds: Crying $\square \quad$ Mumbling

Unusual sensation: Odd smell $\square$ Odd taste

Rising feeling in stomach

visual features

Body numbness

Activity: Normal daily

$$
\text { Hard } \square \quad \text { Light }
$$

Intra ictalsemiology: Blank $\square \quad$ Starring into space

Loss consciousness $\square \quad$ Confused

Color change: Pale Flushing

Breathing change: Noisy

Difficult

Abnormal movement: Jerky $\square$ Twitch $\square$

Posture: Collapsed Stiff $\square$ Floppy $\square$

Got wet Tongue bitten $\square$

Post ictal: Drowzy Tired $\square$ Sleepy

Duration of seizure: Seconds $\square \quad$ Minutes $\square$ Hours

C-EEG finding: Consisten

Inconsistent

D-AEDs taken: Carbamazepine $\square$

Na.Valproate

Lamotrigine $\square$

Oxcarbazepine

Levetiracetam

Phenytoin $\square$

Phenobarbitone

Others 